\title{
Repairing Cyclodialysis by Riveting: A Flanged Polypropylene Suture
}

\author{
Ryosuke Tamiya Masayuki Akimoto \\ Department of Ophthalmology, Osaka Red Cross Hospital, Osaka, Japan
}

\section{Keywords}

Pupillary capture of intraocular lens - Barricading suture · Iris lifting · Intrascleral fixation of intraocular lens · Flanged sutures · Rivets

\begin{abstract}
Cyclodialysis causes intraocular lens (IOL) pupillary capture. It requires surgical repair to prevent this risk of IOL pupillary capture. However, since repairing cyclodialysis is often technically difficult, other methods such as barricading by suturing, pupilloplasty, and pars plana fixation of the IOL were preferred. We applied a double-flanged (riveting) technique for repairing cyclodialysis to prevent IOL pupillary capture following intrascleral fixation of the IOL. Cyclodialysis was surgically repaired by riveting with double-flanged 6-0 polypropylene sutures in 3 cases with no recurrence of pupillary capture during at least 12 months of follow-up. When pupillary capture is related to cyclodialysis, repairing cyclodialysis may help prevent pupillary capture after intrascleral fixation of the IOL. Riveting using a double-flanged 6-0 polypropylene suture could possibly make the procedure simpler and more efficient.
\end{abstract}

\section{Introduction}

Eye blunt trauma causes not only crystalline/intraocular lens (IOL) subluxation/dislocation but also cyclodialysis [1,2]. Repairing cyclodialysis is often technically difficult due to softness and flexibility of the iris and the narrow space between the cornea and lens. Unless cyclodialysis causes hypotony, it may be ignored. However, retraction of the iris plane is thought to be a cause of IOL pupillary capture after suturing or intrascleral fixation of the IOL, which can lead to blurred vision and photopsia [3].

\section{Karger $\stackrel{\text { !' }}{=}$}


Pupillary capture is a possible cause of IOL tilt, chronic uveitis, macular edema, and acute glaucoma [4], resulting in vision loss. Mydriatic or miotic eye drops and laser iridotomy are useful for pupillary capture. In addition, surgery, such as barricading by suturing $[5,6]$ or pupilloplasty [7], is indicated if these treatments were ineffective. However, none of these reported methods for preventing pupillary capture could repair any of the primary causes of iris retraction. Although we have previously reported that repairing cyclodialysis by suturing the iris could prevent pupillary capture [8], it had been still technically difficult. We have devised a novel technique for repairing iridodialysis by riveting with a double-flanged polypropylene suture [9]. We hypothesized that riveting would have also made repair of cyclodialysis easier. Herein, we report 3 cases in which cyclodialysis was repaired by riveting to correct the iris position, consequently preventing IOL pupillary capture.

\section{Surgical Technique}

The surgical technique is shown in online suppl. video (for all online suppl. material, see www.karger.com/doi/10.1159/000518431). A conjunctival incision was made in the direction of the peak of iris capture, which was normally located perpendicular to the haptic of the IOL. The sclera was marked $2.0 \mathrm{~mm}$ and $4.0 \mathrm{~mm}$ posterior to the limbus. Corneal ports were made by $20 \mathrm{G}$ micro vitreoretinal blades in the anterior chamber of the eye in the direction of the mark (Fig. 1A). The anterior chamber was then filled with an ophthalmic viscosurgical device, and the pupillary capture was corrected. Next, a 19.0-mm long ultrathin 30G (LUT30G) needle was inserted parallel to the iris, which lifted the root of the iris and then penetrated the sclera 2.0 $\mathrm{mm}$ posterior to the limbus (Fig. 1B). At first, the needle was placed on the iris to confirm the length. Then, it was inserted behind the iris through the pupil. During proceeding with the needle, the iris was temporally lifted several times to confirm the tip of the needle and amount of the elevation. Finally, the needle pierced the iris root and sclera. A 6-0 polypropylene suture was then inserted into the lumen of the LUT30G needle, which was then removed, leaving a suture behind (Fig. 1C). Approximately $3.0 \mathrm{~cm}$ of this suture was contracted into a small ball by applying high-temperature cautery (Accu-Temp; Beaver Visitec International, Inc., Waltham, MA, USA) (Fig. 1D). The ball was then flattened and widened with a needle holder.

The modified suture was drawn into the anterior chamber (Fig. 1E). The LUT30G needle was then inserted into the sclera to create a tunnel from the mark 4.0-2.0 mm posterior to the limbus (Fig. 1F). The suture was then reinserted in the LUT30G needle, and thereafter the needle was removed, leaving the suture under the scleral tunnel (Fig. 1G). This extra procedure made the position of the flanges away from the limbus where Tenon's capsule was thick enough to cover these flanges. Next, the suture was cut. The end of the suture was then flanged and shortened using cautery until it reached the sclera (Fig. $1 \mathrm{H}$ ). The flanges were not buried in the sclera. The same procedure was repeated on the opposite side.

This study was a hospital-based, single-center case series. We obtained written informed consent from each patient for the original surgery, case details, and any accompanying images published. The procedures used were approved by the Ethics Committee of the Osaka Red Cross Hospital. The study adhered to the tenets of the Declaration of Helsinki.

\section{Case Reports}

The first case was a 65-year-old man who presented to our hospital with double vision in his right eye. Slit-lamp examination revealed a dislocated IOL. Two months earlier, he had undergone intrascleral fixation of an IOL with a 6-mm diameter optic in his right eye.

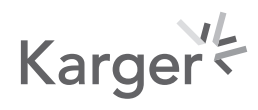




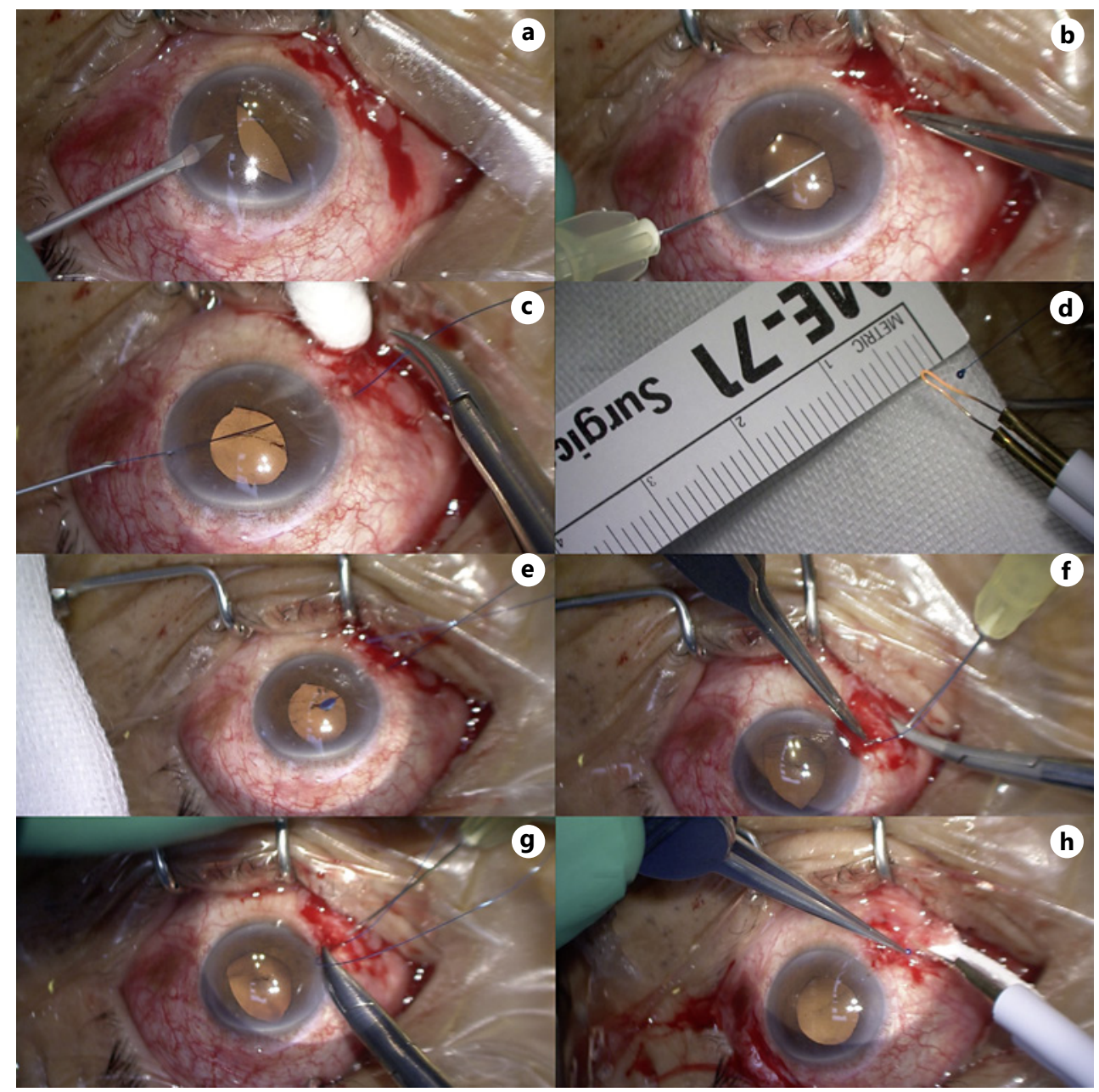

Fig. 1. Procedure of repairing cyclodialysis by the riveting technique. a Corneal ports are made by a 20G micro vitreoretinal blade. b A long ultrathin 30G (LUT30G) needle is inserted parallel to the iris, which lifts the root of the iris and then penetrates the sclera at $2.0 \mathrm{~mm}$ posterior to the limbus. $\mathbf{b}$ A 6-0 polypropylene suture is then inserted into the lumen of the LUT30G needle, which is then removed, leaving the suture behind. $\mathbf{d}$ Approximately $3.0 \mathrm{~cm}$ of the suture is contracted into a small ball by applying high-temperature cautery. e After the ball is flattened, the modified suture is drawn into the anterior chamber. $\mathbf{f}$ The LUT30G needle pierces the sclera to create a tunnel from the mark at 4.0-2.0 $\mathrm{mm}$ posterior to the limbus. $\mathbf{g}$ The suture is again inserted into the LUT30G needle to be placed under the scleral tunnel. $\mathbf{h}$ The end of the suture is flanged and shortened using cautery until it reaches the sclera.

His best-corrected visual acuity (BCVA) was 30/20. The intraocular pressure was $13 \mathrm{~mm} \mathrm{Hg}$. On postoperative day 2 , pupillary capture had been observed. This was fixed using a blunt needle, and thereafter his BCVA was 20/16. The intraocular pressure was $8 \mathrm{~mm} \mathrm{Hg}$. Postoperative slit-lamp examination showed no pupillary capture of the IOL. However, anterior segment optical coherence tomography (AS-OCT) showed cyclodialysis and that the iris had almost captured the IOL (Fig. 2A). Application of miotic eye drops for 2 years had been ineffective (Fig. 2B). Therefore, riveting of the iris was performed. Thereafter, cyclodialysis and distance between the iris and IOL improved (Fig. 2C, D). The position of the iris remained normal during 12 months of follow-up.

The second case was a 59-year-old man who had undergone left-sided cataract surgery 15 years earlier and presented with decreased visual acuity. His BCVA was 20/20, and his 


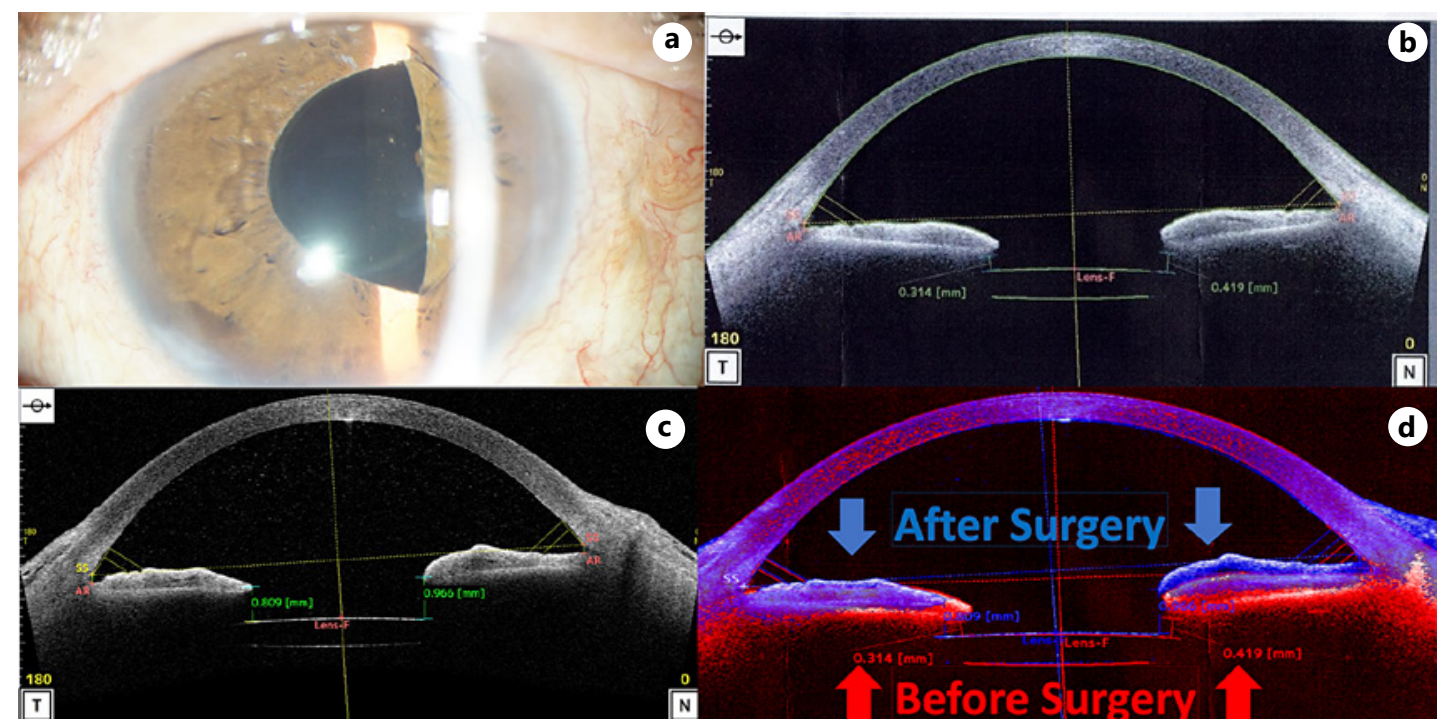

Fig. 2. Case 1. a Slit-lamp photograph showing pupillary capture of the intraocular lens at the first visit. b Exposure of the intraocular lens in the anterior chamber and recession of the iris are shown. $\mathbf{c}$ The position of the iris is corrected by lifting it. $\mathbf{d}$ Position of the iris before (red) and after (blue) surgery.

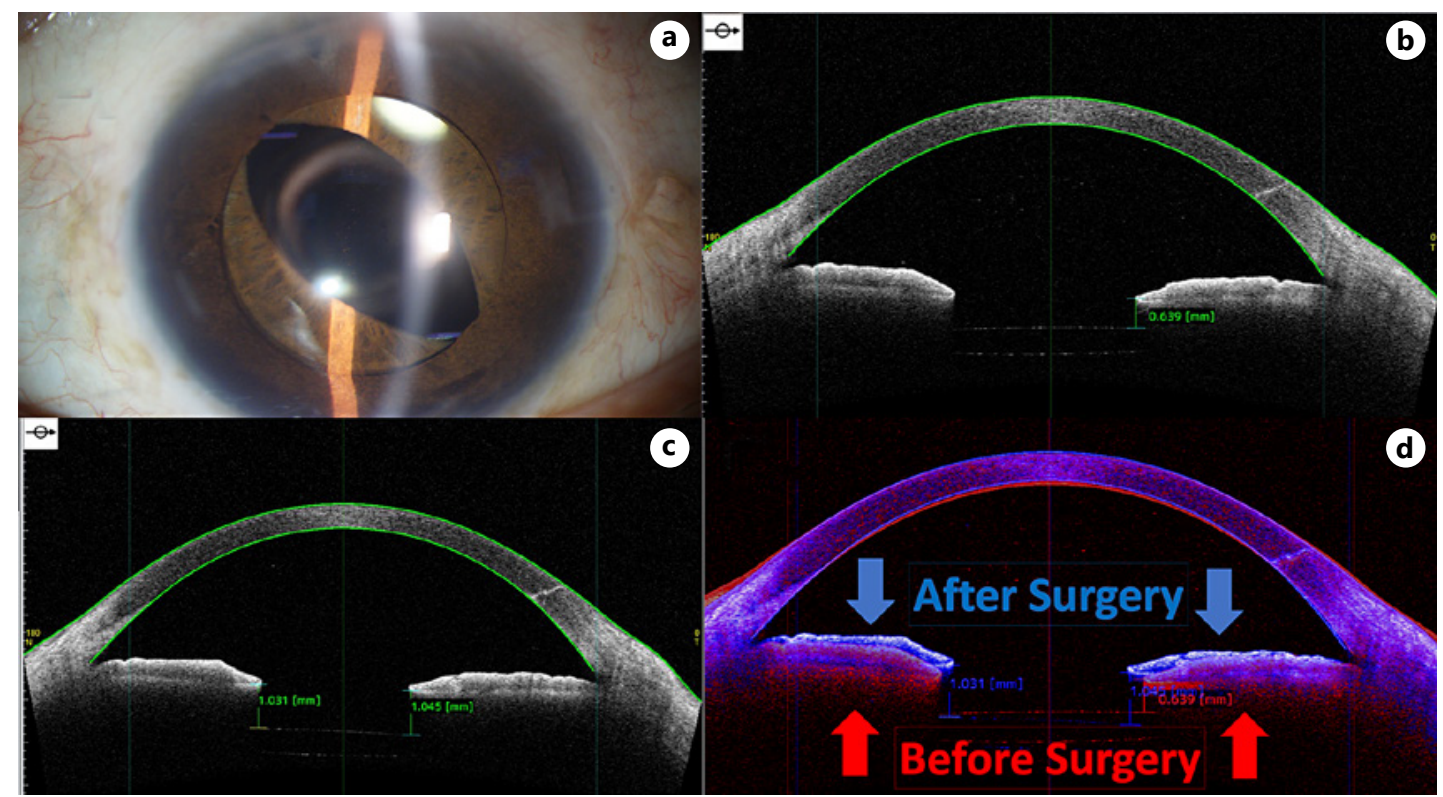

Fig. 3. Case 2. a Slit-lamp photograph showing pupillary capture of the intraocular lens at the first visit. b Exposure of the intraocular lens to the anterior chamber and recession of the iris are shown. c Lifting of the iris to create distance between the iris and intraocular lens. $\mathbf{d}$ Position of the iris before (red) and after (blue) surgery.

intraocular pressure was $14 \mathrm{~mm} \mathrm{Hg}$. Slit-lamp examination showed dislocation of the IOL. The IOL was replaced with another IOL with a 6-mm diameter optic and was intrasclerally fixed. Postoperative slit-lamp examination showed no pupillary capture of the IOL (Fig. 3A). However, AS-OCT of the left eye showed cyclodialysis and that the iris had almost captured the IOL (Fig. 3B). Therefore, we corrected cyclodialysis by riveting the root of the iris. 


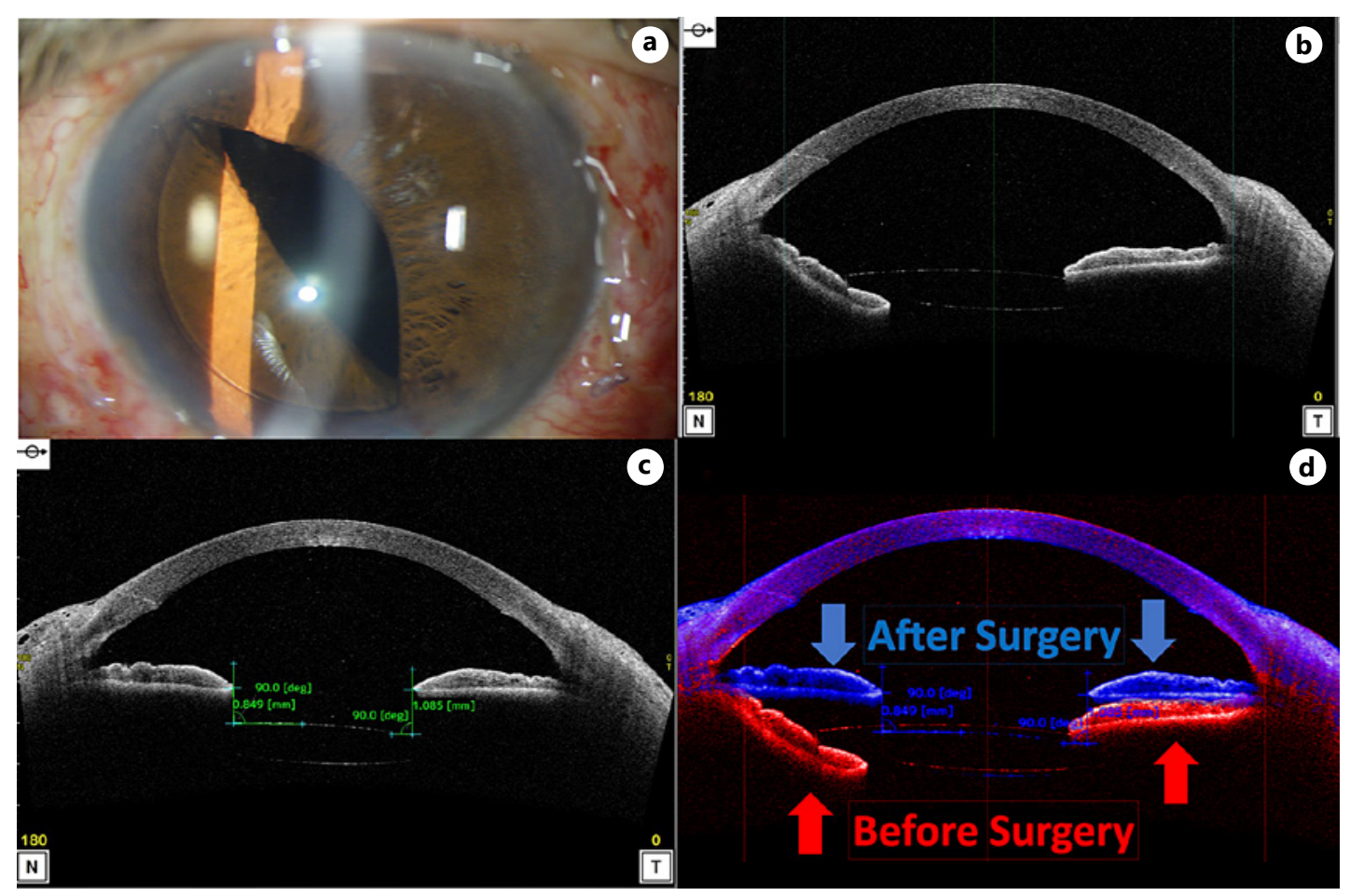

Fig. 4. Case 3. a Slit-lamp photograph obtained at the first visit showing pupillary capture of the intraocular lens. b Bowing of the iris backward toward the intraocular lens. c Position of the iris corrected by lifting it. d Position of the iris before (red) and after (blue) surgery.

Cyclodialysis and distance between the iris and IOL improved after surgery (Fig. 3C, D). No abnormality was found in the iris position during 12 months of follow-up.

The third case was a 59-year-old man who had undergone cataract surgery in his left eye 26 years earlier and presented with decreased visual acuity. His BCVA was 30/20. The intraocular pressure was $14 \mathrm{~mm} \mathrm{Hg}$. Slit-lamp examination showed dislocation of the IOL. This IOL was replaced by another IOL with a 7-mm diameter optic that was intrasclerally fixed. Pupillary capture was observed on postoperative day 2 (Fig. 4A). After discharge, the pupillary capture recurred, and AS-OCT showed cyclodialysis (Fig. 4B). Therefore, we corrected this cyclodialysis by riveting the iris root. Cyclodialysis and distance between the iris and IOL improved postoperatively (Fig. 4C, D). No abnormality was found in the iris position during 12 months of follow-up.

\section{Discussion/Conclusion}

Pupillary capture is a common complication after suturing and intrascleral fixation of an IOL. It seems closely related to a history of trauma in view of existence of cyclodialysis and iridodonesis [10]. Iridodonesis is a risk factor for pupillary capture after suturing and intrascleral fixation of an IOL [11]. Repairing cyclodialysis is often technically difficult due to softness and flexibility of the iris and the narrow space between the cornea and lens. Several techniques for preventing pupillary capture of the IOL have been reported [5-7].

Yoo et al. [5] reported that the $\mathrm{H}$ technique was effective in preventing pupillary capture of intrascleral fixation of the IOL in 40 eyes. After removal of IOL, they created barricades by placing sutures between the iris and IOL with repeat intrascleral fixation of the IOL. Similarly, 
Kim et al. [6] reported the tram-track suture technique. However, these methods may cause pigmentary glaucoma from pigment dispersion as a result of rubbing of the suture against the posterior surface of the iris. Narang and Agarwal [7] used pupilloplasty to prevent pupillary capture by attenuating the pupil size. However, suturing the pupil may obstruct observation of the fundus.

We previously reported that repairing cyclodialysis could restore a normal iris plane. Moreover, separation of the iris and IOL could prevent pupillary capture of the IOL while retaining normal pupillary function [8].

After Yamane et al. [12] reported his flanged intrascleral intraocular lens fixation with the double-needle technique, this technique has become more popularly used instead of suturing. Canabrava et al. $[13,14]$ reported the fixation technique for capsular tension segment: doubleflanged polypropylene suture technique, and IOL repairing using four flanged. We also applied a technique for repairing iridodialysis by riveting with a double-flanged suture [6].

In this case series, we applied the riveting technique for repairing cyclodialysis. We have achieved the best results using LUT30G needles and 6-0 polypropylene sutures. No serious complications have been observed such as massive intraoperative hemorrhage, macular edema, significant inflammation, or out flange exposure up to 12 months of follow-up. In our 3 cases, AS-OCT confirmed successful elevation of the iris plane, preventing repeated pupillary capture.

There were a few complications reported related to flanges such as conjunctival erosion [15] and endophthalmitis [16]. To avoid these complications, we created extra sclera tunnels to introduce the polypropylene suture away from the limbus where Tenon's capsule was thick enough to cover flanges which is described in the Surgical Technique section. Up to 12 months of follow-up, this method might seem contributing to prevention of flange exposure. Although our case series is small in number and limited, repairing angle recession may be a useful method for preventing complications after IOL suturing and intrascleral fixation of IOL, in select cases.

\section{Acknowledgment}

We would like to thank Editage (http://www.editage.com) for editing and reviewing this manuscript for English language.

\section{Statement of Ethics}

This study protocol was reviewed and approved by the Ethics Committee of the Osaka Red Cross Hospital, Approval Reference No. 848. The study adhered to the tenets of the Declaration of Helsinki. We obtained written informed consent from each patient for the original surgery, case details, and any accompanying images published.

\section{Conflict of Interest Statement}

M.A. is a consultant at Kowa Co., Ltd. R.T. has no disclosures to make.

\section{Funding Sources}

No funds, grants, or other support was received.

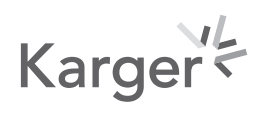


Tamiya and Akimoto: Repairing Cyclodialysis by Riveting

\section{Author Contributions}

All authors attest that they meet the current ICMJE criteria for authorship and approve the final version of the manuscript. R.T. contributed to data interpretation, manuscript writing, editing, and revision. M.A. contributed to concept of the study, patient management, manuscript editing, and revision.

\section{Data Availability Statement}

The data used to support the findings of this study are included within the article.

\section{References}

1 Herschler J. Trabecular damage due to blunt anterior segment injury and its relationship to traumatic glaucoma. Trans Sect Ophthalmol Am Acad Ophthalmol Otolaryngol. 1977;83:239-48.

2 Blanton FM. Anterior chamber angle recession and secondary glaucoma: a study of the aftereffects of traumatic hyphemas. Arch. Ophthalmol. 1964;72:39-43.

3 Cho BJ, Yu HG. Surgical outcomes according to vitreous management after scleral fixation of posterior chamber intraocular lenses. Retina. 2014;34:1977-84.

4 Lindstrom RL, Herman WK. Pupil capture: prevention and management. J Am Intraocul Implant Soc. 1983;9: 201-4.

5 Yoo YJ, Kim ET, Heo JW. Safety barricade suture for preventing pupillary capture of intraocular lens with scleral fixation: H-technique. Retina. 2016;36:206-10.

6 Kim SI, Kim K. Tram-track suture technique for pupillary capture of a scleral fixated intraocular lens. Case Rep Ophthalmol. 2016;7:290-5.

7 Narang P, Agarwal A. Pupilloplasty for pupil size attenuation to prevent pupillary capture: theory of quintet in glued IOL. J Cataract Refract Surg. 2017;43:3-7.

8 Kujime Y, Akimoto M. Repair of angle recession prevents pupillary capture of intrasclerally fixed intraocular lenses. Int Ophthalmol. 2019;39:1163-8.

9 Kusaka M, Miyamoto N, Akimoto M. Repairing iridodialysis by riveting with a double-flanged polypropylene suture. J Cataract Refract Surg. 2019;45:1531-4.

10 Loo AV, Lai JS, Tham CC, Lam DS. Traumatic subluxation causing variable position of the crystalline lens. J Cataract Refract Surg. 2002;28:1077-9.

11 Bang SP, Joo CK, Jun JH. Reverse pupillary block after implantation of a scleral-sutured posterior chamber intraocular lens: a retrospective, open study. BMC Ophthalmol. 2017;17:35.

12 Yamane S, Sato S, Maruyama-Inoue M, Kadonosono K. Flanged intrascleral intraocular lens fixation with double-needle technique. Ophthalmology. 2017;124:1136-42.

13 Canabrava S, Bernardino L, Batisteli T, Lopes G, Diniz-Filho A. Double-flanged-haptic and capsular tension ring or segment for sutureless fixation in zonular instability. Int Ophthalmol. 2018;38:2653-62.

14 Canabrava S, Canêdo Domingos Lima AC, Ribeiro G. Four-flanged intrascleral intraocular lens fixation technique: no flaps, no knots, no glue. Cornea. 2020;39:527-8.

15 Canabrava S, Andrade N Jr, Rezende PH. Scleral fixation of a 4-eyelet foldable intraocular lens in patients with aphakia using a 4-flanged technique. J Cataract Refract Surg. 2020;47:265-9.

16 Roditi E, Brosh K, Assayag E, Weill Y, Zadok D. Endophtalmitis associated with flange exposure after a 4-flanged canabrava fixation technique. JCRS Online Case Rep. 2021;9:e00042-5. 